\title{
A THEOREM ON THE GROWTH OF ENTIRE FUNCTIONS ON ASYMPTOTIC PATHS AND ITS APPLICATION TO THE OSCILLATION THEORY OF $\boldsymbol{w}^{\prime \prime}+A \boldsymbol{w}=0$
}

Dedicated to Professor Nobuyuki Suita on the occasion of his sixtieth birthday

By NoBUSHIGE TODA

\section{Introduction.}

Let $A=A(z)$ be a transcendental entire function and let $w_{1}, w_{2}$ be two linearly independent entire solutions of the differential equation

$$
w^{\prime \prime}+A w=0 .
$$

It is known that any non-zero solution of (1) is an entire function of infinite order $([1])$. Put

$$
E=w_{1} w_{2}
$$

It then holds ([1], p. 354) that

$$
4 A=\left(E^{\prime} / E\right)^{2}-2 E^{\prime \prime} / E-(c / E)^{2},
$$

where $c$ is the Wronskian of $w_{1}$ and $w_{2}$, which is a non-zero constant in this case.

For an entire function $f$ we denote the order of $f$ by $\rho(f)$, the lower order of $f$ by $\mu(f)$ and the order of $N(r, 1 / f)$ by $\lambda(f)$.

S. B. Bank and I. Laine ([1], Theorem 2, (A)) proved from (2) that $\rho(A)<$ $1 / 2$ implies $\lambda(E)=+\infty$. They also gave examples of (1) with two linearly independent entire solutions each having no zeros, in each case of which, $\rho(A)$ is either a positive integer or $+\infty$ ([1], p. 356).

It is conjectured that if $\rho(A)$ is finite and not a positive integer, then we always have $\lambda(E)=+\infty$ (see [2], p. 164). In this direction J. Rossi ([12]) and L.-C. Shen ([13]) proved some results which contains that $\rho(A) \leqq 1 / 2$ implies $\lambda(E)=+\infty$. Recently, C.-Z. Huang ([9]) proved the following result which generalizes them.

THEOREM A. If $\mu(A)<1$, then either $\lambda(E)=+\infty$ or

Received September 30, 1992. 


$$
\mu(A)^{-1}+\lambda(E)^{-1} \leqq 2 \quad([9], \text { Theorem } 1) .
$$

One of our main purpose of this paper is to give a result which contains Theorem A. To prove it, we need a growth property of $A(z)$ in the set

$$
\{z:|A(z)|>1\}
$$

and so we shall first give a result on the growth of entire functions along asymptotic paths. We shall assume that the reader is familiar with the satndard notation of the Nevanlinna theory of meromorphic functions ([5]).

\section{Growth of entire functions along asymptotic paths.}

A few years ago J. Rossi and A. Weitsman ([11]) proved the following.

TheOREM B. Let $f(z)$ be a transcendental entire function. Suppose that for some constant $K$ the set

$$
\{z:|f(z)|>K\}
$$

contains at least two components. Then there exists a path $\Gamma$ from 0 to $\infty$ such that for $z \varepsilon \Gamma$

$$
\log |f(z)|>|z|^{\rho(f) /(2 \rho(f)-1)-\varepsilon(z)} \quad(0 \leqq \varepsilon(z) \rightarrow 0 \text { as } z \rightarrow \infty) .
$$

(We consider $\rho(f) /(2 \rho(f)+k)=1 / 2$ when $\rho(f)=+\infty$ and $k$ is finite.)

Examples showing that Theorem $B$ is sharp are given in [4]. Besides this result we can find interesting results on the growth of entire and subharmonic functions along asymptotic paths ([3], [4], [10], [11], [14], [15] and Chapter 8 in [8]).

The purpose of this section is to improve Theorem $B$ and to give a subharmonic analogue, which is an improvement of Theorem 1 in [4].

\section{2-1. Lemmas.}

We shall give some lemmas for later use. Let $D$ be an unbounded regular plane domain. We put

and

$$
E(r)=\left\{\theta \varepsilon[0,2 \pi): r e^{i \theta} \varepsilon D\right\}
$$

$$
\theta(r)=\left\{\begin{array}{l}
+\infty \quad \text { if } \quad\{|z|=r\} \subset D \\
\text { the measure of } E(r) \text { otherwise. }
\end{array}\right.
$$

It is clear that there is a positive number $a$ such that $\theta(r)>0$ for all $r \geqq a$.

LEMMA 1. If

$$
\liminf _{r \rightarrow \infty}(\log r)^{-1} \pi \int_{a}^{r} \frac{d t}{t \theta(t)}=\mu \quad(1 / 2 \leqq \mu<\infty)
$$


then there exists $u>0$ harmonic in $D$ such that for all $z \varepsilon D$

$$
u(z) \geqq|z|^{\mu-\varepsilon(z)} \quad(0 \leqq \varepsilon(z) \rightarrow 0 \text { as } z \rightarrow \infty)
$$

([11], Lemma 1 and its correction).

Lemma 2. Let $g(z)$ be regular in $D$ and continuous on the closure of $D$ such that

$$
|g(z)| \leqq 1 \quad(z \varepsilon \partial D) .
$$

If there exists one point $z_{0}$ in $D$ such that

then

$$
\left|g\left(z_{0}\right)\right|>1
$$

$$
\log \log M(r, g) \geqq \pi \int_{a}^{r / 2} \frac{d t}{t \theta(t)}+O(1),
$$

where $M(r, g)=\sup \{|g(z)|:(|z|=r) \cap D\} \quad([17]$, p. 117).

LEMMA 3. Let $v(z)$ be a non-constant subharmonic function in $|z|<\infty$. Then there exists $a$ path $\Gamma$ tending to $\infty$ such that

$$
v(z) \longrightarrow+\infty \text { as } z \longrightarrow \infty \text { on } \Gamma
$$

([14], Theorem 1).

\section{2-2. Theorem.}

We shall give a result generalizing Theorem $B$.

THEOREM 1. Let $f(z)$ be a transcendental entire function with $\mu(f)<+\infty$. Suppose that for some constant $K$ the set

$$
\{z:|f(z)|>K\}
$$

contains at least $N$ components $D_{1}, \cdots, D_{N}$, where $N \geqq 2$. Then for each $j(=1, \cdots$, $N)$ there exists a path $\Gamma$, tending to $\infty$ in $D$, such that on $\Gamma_{\text {, }}$

$$
\log |f(z)|>|z|^{\rho(f) /(2 \rho(f)+1-N)-\varepsilon_{j}(z)} \quad\left(0 \leqq \varepsilon_{j}(z) \rightarrow 0 \text { as } z \rightarrow \infty\right) .
$$

Proof. It is clear that $D_{1}, \cdots, D_{N}$ are mutually disjoint unbounded regular domains in $|z|<\infty$ and there exists an $a>0$ such that for all $r \geqq a$

$$
\{|z|=r\} \cap D, \neq \phi \quad(\jmath=1, \cdots, N) .
$$

We here use $\theta_{j}(r)$ for $D$, instead of $\theta(r)$ defined for $D$ in (4). Then

and

$$
\theta_{j}(r)>0 \quad(r \geqq a)
$$




$$
\sum_{j=1}^{N} \theta_{j}(r) \leqq 2 \pi
$$

From (6) we obtain the inequality

$$
\sum_{j=1}^{N} \int_{a}^{r} \frac{\theta_{j}(t)}{t} d t \leqq 2 \pi \log \frac{r}{a}
$$

and by the Cauchy-Schwarz inequality we have

$$
\int_{a}^{r} \frac{\theta_{j}(t)}{t} d t \int_{a}^{r} \frac{d t}{t \theta_{j}(t)} \geqq\left(\int_{a}^{r} \frac{d t}{t}\right)^{2}=\left(\log \frac{r}{a}\right)^{2}
$$

From (7) and (8) we have

$$
\sum_{j=1}^{N} \frac{1}{\{\log (r / a)\}^{-1} \pi \int_{a}^{r} \frac{d t}{t \theta_{j}(t)}} \leqq 2 .
$$

Applying Lemma 2 to $f(z) / K$ in $D$, we obtain the following inequalities :

$$
\log \log M(2 r, f) \geqq \pi \int_{a}^{r} \frac{d t}{t \theta_{j}(t)}+O(1)
$$

from which we have

$$
\liminf _{r \rightarrow \infty}(\log r)^{-1} \pi \int_{a}^{r} \frac{d t}{t \theta_{j}(t)} \leqq \mu(f)<+\infty .
$$

From (9) and (10) we have for each $j(=1, \cdots, N)$

$$
\frac{N-1}{\{\log (r / a)\}^{-1}\{\log \log M(2 r, f)+O(1)\}}+\frac{1}{\{\log (r / a)\}^{-1} \pi \int_{a}^{r} \frac{d t}{t \theta_{j}(t)}} \leqq 2
$$

and hence

$$
\frac{N-1}{\rho(f)}+\frac{1}{\liminf _{r \rightarrow \infty}(\log r)^{-1} \pi \int_{a}^{r} \frac{d t}{t \theta_{j}(t)}} \leqq 2 .
$$

From (11) and (12) we have for each $j(=1, \cdots, N)$

$$
\frac{\rho(f)}{2 \rho(f)+1-N} \leqq \liminf _{r \rightarrow \infty}(\log r)^{-1} \pi \int_{a}^{r} \frac{d t}{t \theta_{j}(t)} \leqq \mu(f)<+\infty
$$

Since $\rho(f) /(2 \rho(f)+1-N) \geqq 1 / 2$ in (13), there exists a positive harmonic function $u$, in $D$, such that for all $z \in D$,

$$
u_{j}(z) \geqq|z|^{\rho(f) /(2 \rho(f)+1-N)-\varepsilon_{0}(z)} \quad\left(0 \leqq \varepsilon_{0}(z) \rightarrow 0 \text { as } z \rightarrow \infty\right)
$$

by Lemma 1 . We can find $z$, in $D$, for which 


$$
\left|f\left(z_{j}\right)\right|>K
$$

and choose a positive constant $\delta$ so small that

We then define

$$
\log \left|f\left(z_{\jmath}\right)\right|>\delta u_{j}\left(z_{j}\right)+\log K \text {. }
$$

$$
U_{j}(z)= \begin{cases}\max \left\{\log (|f(z)| / K)-\delta u_{j}(z), 0\right\} & \left(z \in D_{j}\right) \\ 0 & \left(z \notin D_{j}\right) .\end{cases}
$$

Since $U_{j}\left(z_{j}\right)>0$ and $U_{j}(z)=0$ for $z \notin D_{\jmath}$, it is clear that $U_{j}(z)$ is a non-constant subharmonic function in $|z|<\infty$. Hence by Lemma 3 there exists a path $\Gamma_{\text {J }}$ tending to $\infty$ such that

$$
U_{j}(z) \longrightarrow+\infty \text { as } z \longrightarrow \infty \text { on } \Gamma_{\jmath} .
$$

We may assume without loss of generality that

$$
U_{j}(z)>0 \text { on } \Gamma_{j}
$$

so that $\Gamma_{\mathrm{g}}$ lies in $D_{\mathrm{\jmath}}$ and on $\Gamma_{\mathrm{\jmath}}$

$$
U_{j}(z)=\log |f(z)|-\delta u_{j}(z)-\log K>0 .
$$

Thus we have by (14)

$$
\log |f(z)|>|z|^{\rho(f) /(2 \rho(f)+1-N)-\varepsilon_{j}(z)} \quad\left(0 \leqq \varepsilon_{j}(z) \rightarrow 0 \text { as } z \rightarrow \infty\right) \text { on } \Gamma_{J} .
$$

Remark 1. By a well-known Ahlfors' theorem (see [6], p. 255), it is known that $N=1$ when $\mu(f)<1$ and $N \leqq 2 \mu(f)$ when $1 \leqq \mu(f)<+\infty$.

From (9) and (10) we obtain for $r \geqq a$

$$
N \leqq 2\{\log \log M(2 r, f)+O(1)\} / \log (r / a),
$$

which reduces to $N \leqq 2 \mu(f)$ when $N \geqq 2$.

Example 1. Let

Then,

$$
f(z)=\cos h z^{N / 2} \quad(N=2,3, \cdots) .
$$

$$
M(r, f)=\frac{\exp \left(r^{N / 2}\right)+\exp \left(-r^{N / 2}\right)}{2} \text { and } \rho(f)=\mu(f)=N / 2 .
$$

It is easily seen that for $k=0,1, \cdots, N-1$ and for $0 \leqq t<+\infty$

and

$$
\left|f\left(t e^{(2 k+1) \pi \imath / N}\right)\right| \leqq 1
$$

$$
\log \left|f\left(t e^{2 k \pi \imath / N}\right)\right|>t^{N / 2-\varepsilon(t)} \quad(0<\varepsilon(t) \rightarrow 0 \quad \text { as } t \rightarrow+\infty) .
$$

Remark 2. This example shows that Theorem 1 is sharp. 
Example 2. Let $f(z)$ be an entire function of finite lower order with $N(\geqq 2)$ distinct finite asymptotic values. Then, for a sufficiently large $K$ the set

$$
\{z:|f(z)|>K\}
$$

has at least $N$ components.

We can find a concrete example of $f(z)$ with $N$ distinct finite asymptotic values in [8], p. 562 .

\section{2-3. Subharmonic analogue.}

Let $v(z)$ be a non-constant subharmonic function in $|z|<\infty$. Put

$$
\begin{gathered}
B(r, v)=\sup _{|z|=r} v(z), \\
\left.\rho=\limsup _{r \rightarrow \infty} \log B(r, v) / \log r \quad \text { (the order of } v\right), \\
\left.\mu=\liminf _{r \rightarrow \infty} \log B(r, v) / \log r \quad \text { (the lower order of } v\right) .
\end{gathered}
$$

It is said that $v(z)$ has at least $N$ tracts in $|z|<\infty$ if and only if

$$
\{z: v(z)>K\}
$$

has at least $N$ components for all sufficiently large $K$, where $N$ is a positive integer ([7], [8]). When $N \geqq 2$, the following result is given ([8], p. 593).

THEOREM C. Suppose that $v(z)$ has at least $N(\geqq 2)$ tracts in the finite plane. Then there exist sectionally polygonal paths $\gamma_{1}, \cdots, \gamma_{N}$ from 0 to $\infty$ such that

1) $\gamma_{j} \cap \gamma_{k}=\{0, \infty\} \quad(j \neq k)$,

2) $\gamma_{\jmath}$ and $\gamma_{\jmath+1}$ bound a domain $D_{\jmath}$ and $D_{j} \cap \gamma_{k}=\phi\left(\gamma_{N+1}=\gamma_{1}\right)$,

3) $v(z)$ is bounded on the $\gamma_{3}$ and not bounded above in the $D_{\jmath}$.

Put

$$
B_{j}(r, v)=\sup \left\{v(z):(|z|=r) \cap D_{j}\right\} .
$$

By 3) in Theorem C, there exists $z_{j} \in D$, for each $j$ such that

$$
v\left(z_{j}\right)>0
$$

and for all sufficiently large $r$

$$
B_{j}(r, v)>0
$$

Further there exists a positive number $M$ such that

$$
V(z)=v(z)-M
$$

is negative on $\gamma_{1} \cup \cdots \cup \gamma_{N}$.

LEMMA 4. $\log B_{j}(r, v) \geqq \pi \int_{1}^{r / 2} \frac{d t}{t \theta_{j}(t)}+O(1)$ 
where we use $\theta_{j}(r)$ for $D$, instead of $\theta(r)$ defined for $D$ in (4).

We can prove this lemma by applying Theorem 8.3 ([8], p. 548) to $V_{\jmath(z)}=$ $\max \left\{V_{(z)}, 0\right\}$ if $z \in D_{\jmath},=0$ otherwise.

THEOREM 2. Suppose that $v(z)$ has at least $N(\geqq 2)$ tracts in the finite plane and $\mu<+\infty$. Then there exists a path $\Gamma_{\text {J }}$ tending to $\infty$ in $D$, such that

$$
v(z)>|z|^{\rho /(2 \rho+1-N)-\varepsilon_{j}(z)} \quad\left(0 \leqq \varepsilon_{j}(z) \rightarrow 0 \text { as } z \rightarrow \infty\right)
$$

on $\Gamma_{j}(j=1, \cdots, N)$.

We can prove this theorem as in the case of Theorem 1 using Lemma 4 instead of Lemma 2. We note that $N \leqq 2 \mu$ as in Remark 1 .

\section{Application to the oscillation theory of $w^{\prime \prime}+A w=0$.}

We shall first give some lemmas for later use. We use the same notation as in the section 1 .

LEMMA 5. If $\rho(E)<+\infty$, for a given $\varepsilon>0$ there exists a positive number $d=d(\varepsilon)$ such that

$$
\left|\left(E^{\prime} / E\right)^{2}\left(r e^{i \theta}\right)-2\left(E^{\prime \prime} / E\right)\left(r e^{i \theta}\right)\right| \leqq r^{d}
$$

for all $r \geqq r_{0}>1$ and all $\theta \notin J(r)$, where the angular measure of $J(r), m(J(r)) \leqq \varepsilon \pi$ ([12], Lemma 1).

LEMMA 6. If $\lambda(E)<\rho(E)$, then

$$
\mu(E)=\rho(E)=\mu(A)=\rho(A)
$$

and these numbers are equal to an integer or $+\infty$.

Proof. From (2) we easily have

$$
2 T(r, E)=2 N(r, 1 / E)+T(r, A)+S(r, E) .
$$

Set

$$
E(z)=\Pi(z) e^{P(z)},
$$

where $\Pi(z)$ is the Weierstrass product of the zeros of $E$ and $P(z)$ is an entire function. Then, it is known that $\rho(\Pi)=\lambda(E)$ (see [5]).

a) The case $\rho(E)=+\infty$. In this case, $P(z)$ is transcendental and it is easy to see that $\mu(E)=+\infty$. Let $\alpha$ be any number such that $\lambda(E)<\alpha<+\infty$. Then from (15) we have

$$
2 T_{\alpha}(r, E)=2 N_{\alpha}(r, 1 / E)+T_{\alpha}(r, A)+S_{\alpha}(r, E),
$$

where 


$$
\begin{aligned}
& T_{\alpha}(r, E)=\int_{1}^{r} \frac{T(t, E)}{t^{1+\alpha}} d t \text { is of lower order }+\infty, \\
& N_{\alpha}(r, 1 / E)=\int_{1}^{r} \frac{N(t, 1 / E)}{t^{1+\alpha}} d t \text { is bounded, } \\
& T_{\alpha}(r, A)=\int_{1}^{r} \frac{T(t, A)}{t^{1+\alpha}} d t \leqq T(r, A) / \alpha
\end{aligned}
$$

and

$$
S_{\alpha}(r, E)=\int_{1}^{r} \frac{S(t, E)}{t^{1+\alpha}} d t=o\left(T_{\alpha}(r, E)\right) \quad(r \rightarrow \infty)
$$

(see [16], Proposition 1 and Lemma 1), so that

$$
+\infty=\liminf _{r \rightarrow \infty} \frac{\log T_{\alpha}(r, E)}{\log r}=\liminf _{r \rightarrow \infty} \frac{\log T_{\alpha}(r, A)}{\log r} \leqq \mu(A) .
$$

We have $\mu(A)=\rho(A)=+\infty$.

b) The case $\rho(E)<+\infty$. In this case, $P(z)$ must be a polynomial and it is easy to see that

$$
\mu(E)=\rho(E)=\text { the degree of } P(z)
$$

since $\lambda(E)<\rho(E)$. From (15) and (17) we have

$$
\mu(A)=\rho(A)=\mu(E)=\rho(E)=\text { an integer. }
$$

THEOREM 3. Suppose that $\mu(A)<+\infty$ and for a positive constant $K$ not smaller than $1 / 2$ the set

$$
\{z:|A(z)|>K\}
$$

has at least $N$ components. Then, either $\rho(E)=+\infty$ or

$$
\frac{N}{\mu(A)}+\frac{1}{\rho(E)} \leqq 2 .
$$

Proof. Suppose that $\rho(E)<+\infty$. Let $D_{0}$ be a component of the set

$$
\{z:|E(z)|>|c|\},
$$

which is a non-empty unbounded set since $E$ is transcendental by (2).

The set $\{z:|A(z)|>K\}$ has at least $N$ components, and since $A(z)$ is transcendental and Theorem 1 holds for $N \geqq 2$, for any positive integer $p$ and for $K_{1}=\max \{K, M(1, A)\}$ the set

$$
\left\{z: \log |A(z)|-p \log |z|-\log K_{1}>0\right\}
$$

has at least $N$ unbounded components. Let $D_{1}, \cdots, D_{N}$ be those $N$ unbounded components. For $\jmath=0,1, \cdots, N$, put 
and

$$
E_{j}(r)=\left\{\theta \in[0,2 \pi): r e^{i \theta} \leqq D_{j}\right\}
$$

$$
\theta_{j}(r)=\left\{\begin{array}{l}
+\infty \text { if }\{|z|=r\} \subset D_{\jmath} \\
\text { the measure of } E_{j}(r) \text { otherwise. }
\end{array}\right.
$$

Then there is a positive number $a$ such that $\theta_{j}(r)>0$ for all $r \geqq a$ and for all $j$. By Lemma 2 we have

$$
\log \log M(r, E) \geqq \pi \int_{a}^{r / 2} \frac{d t}{t \theta_{0}(t)}+O(1)
$$

and

$$
\log \left\{\log M(r, A)-p \log r-\log K_{1}\right\} \geqq \pi \int_{a}^{r / 2} \frac{d t}{t \theta_{j}(t)} O(1)
$$

for $j=1, \cdots, N$.

For any fixed positive number $\varepsilon<1$, let $p$ be a positive integer such that $p>d$, where $d$ is the constant given in Lemma 5 . We define for $\jmath=0,1, \cdots, N$

$$
l_{j}(t)=\left\{\begin{array}{l}
2 \pi \text { if } \theta_{j}(t)=+\infty \\
\theta_{j}(t) \text { otherwise }
\end{array}\right.
$$

Then applying Lemma 5 to (2) we obtain the inequality

$$
\sum_{j=0}^{N} l_{j}(t) \leqq(2+\varepsilon) \pi
$$

for all $r \geqq b=\max \left(a, r_{0}\right)$ from which we have

$$
\sum_{j=0}^{N} \int_{b}^{r} \frac{l_{j}(t)}{t} d t \leqq(2+\varepsilon) \pi \log (r / b) .
$$

By the Cauchy-Schwarz inequality

$$
\int_{b}^{r} \frac{l_{j}(t)}{t} d t \int_{b}^{r} \frac{d t}{t l_{j}(t)} \geqq\left(\int_{b}^{r} \frac{d t}{t}\right)^{2}=\left(\log \frac{r}{b}\right)^{2} .
$$

From (21) and (22) we obtain the inequality

Define

$$
\sum_{j=0}^{N} \frac{\log (r / b)}{\pi \int_{b}^{r} \frac{d t}{t l_{j}(t)}} \leqq 2+\varepsilon
$$

$$
B_{0}=\left\{r: \theta_{0}(r)=+\infty\right\} .
$$

Then, $B_{0}$ is a sum of intervals. Let

$$
\chi_{0}(r)= \begin{cases}1 & \text { if } r \text { belongs to } B_{0} \\ 0 & \text { otherwise }\end{cases}
$$


If $r$ belongs to $B_{0}$ and $r \geqq b$, we have

and

$$
\theta_{j}(r)=l_{j}(r) \quad \text { for } \quad j=1, \cdots, N
$$

$$
\theta_{1}(r)+\cdots+\theta_{N}(r) \leqq \varepsilon \pi
$$

from (20). 'Thus, if we set

then

$$
F_{\jmath}=\left\{r ; \theta_{\jmath}(r) \leqq \varepsilon \pi\right\},
$$

$$
B_{0} \subset \bigcup_{\jmath=1}^{N} F_{\jmath}
$$

Define

and put

$$
\phi_{j}(r)= \begin{cases}1 & \text { if } r \text { belongs to } F \\ 0 & \text { otherwise }\end{cases}
$$

$$
M(r)=\log M(r, A)-p \log r-\log K_{1}
$$

We then have from (24)

$$
\int_{b}^{r} \frac{\chi_{0}(t)}{t} d t \leqq \sum_{j=1}^{N} \int_{b}^{r} \frac{\phi_{j}(t)}{t} d t \leqq N \varepsilon \log M(2 r)+O(1)
$$

since $\varepsilon^{-1} \phi_{j}(t) \leqq \pi / \theta_{j}(t)$ and so

$$
\varepsilon^{-1} \int_{b}^{r} \frac{\phi_{j}(t)}{t} d t \leqq \pi \int_{b}^{r} \frac{d t}{t \theta_{j}(t)} \leqq \log M(2 r)+O(1)
$$

by (19).

(i) The case $N \geqq 2$. In this case it is clear that for $j=1, \cdots, N$

Since

$$
0<\theta_{j}(r)<2 \pi \text { and } \theta_{j}(r)=l_{j}(r) \quad(r \geqq b) .
$$

$$
\pi \int_{b}^{r} \frac{d t}{t \theta_{0}(t)}=\pi \int_{b}^{r} \frac{d t}{t l_{0}(t)}-\frac{1}{2} \int_{b}^{r} \frac{\chi_{0}(t)}{t} d t
$$

from (18), (19), (23) and (25) we obtain for $r \geqq b$

$$
\frac{N \log (r / b)}{\log M(2 r)+O(1)}+\frac{\log (r / b)}{\log \log M(2 r, E)+(N \varepsilon / 2) \log M(2 r)+O(1)} \leqq 2+\varepsilon .
$$

Let $\left\{r_{n}\right\}$ be a sequence tending to $+\infty$ such that

$$
\lim _{n \rightarrow \infty} \frac{\log \log M\left(2 r_{n}, A\right)}{\log 2 r_{n}}=\mu(A) .
$$

Put $r=r_{n}$ in (27) and let $n$ tend to $+\infty$. We then obtain 


$$
\frac{N}{\mu(A)}+\frac{1}{\rho(E)+N \varepsilon \mu(A) / 2} \leqq 2+\varepsilon .
$$

Tending $\varepsilon \rightarrow 0$, we have

$$
\frac{N}{\mu(A)}+\frac{1}{\rho(E)} \leqq 2 .
$$

(ii) The case $N=1$. Let

$$
B_{1}=\left\{r: \theta_{1}(r)=+\infty\right\} .
$$

Then, $B_{1}$ is a sum of intervals. Define

$$
\chi_{1}(r)= \begin{cases}1 & \text { if } r \text { belongs to } B_{1} \\ 0 & \text { otherwise }\end{cases}
$$

If $r$ belongs to $B_{1}$ and $r \geqq b$, we have

by (20). Put

$$
\theta_{0}(r) \leqq \varepsilon \pi
$$

and

$$
F_{0}=\left\{r: \theta_{0}(r) \leqq \varepsilon \pi\right\}
$$

We then have

$$
\psi_{0}(r)= \begin{cases}1 & \text { if } r \text { belongs to } F_{0} \\ 0 & \text { otherwise }\end{cases}
$$

$$
\int_{b}^{r} \frac{\chi_{1}(t)}{t} d t \leqq \int_{b}^{r} \frac{\varphi_{0}(t)}{t} d t \leqq \varepsilon \log \log M(2 r, E)+O(1)
$$

since $B_{1} \subset F_{0}, \varepsilon^{-1} \psi_{0}(t) \leqq \pi / \theta_{0}(t)$ and so

$$
\varepsilon^{-1} \int_{b}^{r} \frac{\phi_{0}(t)}{t} d t \leqq \pi \int_{b}^{r} \frac{d t}{t \theta_{0}(t)} \leqq \log \log M(2 r, E)+O(1)
$$

by (18). Since

$$
\pi \int_{b}^{r} \frac{d t}{t \theta_{1}(t)}=\pi \int_{b}^{r} \frac{d t}{t l_{1}(t)}-\frac{1}{2} \int_{b}^{r} \frac{\chi_{1}(t)}{t} d t
$$

from (18), (19), (23) and (25) for $N=1,(26)$ and (29), we have

$$
\begin{aligned}
& \frac{\log (r / b)}{\log M(2 r)+\varepsilon \log \log M(2 r, E)+O(1)} \\
& +\frac{\log (r / b)}{\log \log M(2 r, E)+(\varepsilon / 2) \log M(2 r)+O(1)} \leqq 2+\varepsilon .
\end{aligned}
$$

Then as in the case of $N \geqq 2$ where we obtained (28) from (27), we obtain the inequality 


$$
-\frac{1}{\mu(A)}+\frac{1}{\rho(E)} \leqq 2
$$

from (30).

CORLLARY. Under the same assumption as in Theorem 3,

1) If $\mu(A)<\rho(A)=+\infty$, then $\lambda(E)=+\infty$.

2) When $\rho(A)<+\infty$, if $\mu(A)<\rho(A)$ or if $A$ is of regular growth and $\rho(A)$ is not equal to an integer, then either $\lambda(E)=+\infty$ or

$$
\frac{N}{\mu(A)}+\frac{1}{\lambda(E)} \leqq 2 \text {. }
$$

3) If $\mu(A) \leqq 1 / 2$ or if $\mu(A)=N / 2$ in case of $N \geqq 2$, then

$$
\lambda(E)=+\infty \text {. }
$$

Proof. 1) We easily have

$$
\rho(A) \leqq \rho(E)
$$

from (15) and since $\mu(A)<\rho(A)=+\infty$ we have

by Lemma 6 .

$$
\lambda(E)=\rho(E)=+\infty
$$

2) In this case, we have

$$
\lambda(E)=\rho(E)
$$

by Lemma 6 . We obtain (31) from Theorem 3.

3) Noting the fact that

$$
\text { " } N=1 \text { if } \mu(A)<1 \text { and } N \leqq 2 \mu(A) \text { if } 1 \leqq \mu(A)<+\infty \text { " }
$$

(see Remark 1), we easily obtain $\lambda(E)=+\infty$ when $\mu(A) \leqq 1 / 2$ or $\mu(A)=N / 2$ in case $N$ is odd from 2) of this corollary.

When $N$ is even and positive, $\mu(A)=N / 2$ implies $\rho(E)=+\infty$ by Theorem 3. If $\lambda(E)<+\infty$, then $\mu(A)=\rho(E)=+\infty$ by Lemma 6 . This is a contradiction. $\lambda(E)$ must be equal to $+\infty$.

Remark 3. The functions of Examples 1 and 2 in the section 2 satisfy the conditions of Theorem 3 for $N \geqq 2$.

\section{REFERENCES}

[1] S. B. BANK AND I. LAINE, On the oscillation theory of $f^{\prime \prime}+A f=0$ where $A$ is entire, Trans. Amer. Math. Soc. 273-1 (1982), 351-363. 
[2] S.B. BANK AND J.K. LANGLEy, Oscillation theory for higher order linear differential equations with entire coefficients, Complex Variables 16 (1991), 163175.

[3] K.-H. CHANG, Asymptotic values of entire and meromorphic functions, Sci. Sinica 20-6 (1977), 720-739.

[4] A.E. EREMENKo, Growth of entire functions and subharmonic functions on asymptotic curves, Siberian Math. J. 21 (1981), 673-683.

[5] W.K. HaYman, Meromorphic functions, Oxford at the Clarendon Press (1964).

[6] W.K. HAYMAN, On the growth of integral functions on asymptotic paths, J. London Math. Soc. 24 (1960), 251-264.

[7] W.K. Hayman and P. B. Kennedy, Subharmonic functions, Vol. 1, Academic Press, London (1976).

[8] W. K. Hayman, Subharmonic functions, Vol. 2, Academic Press, London (1989).

[9] C.-Z. HuANG, Some results on the complex oscillation theory of second order linear differential equations, Kodai Math. J. 14 (1991), 313-319.

[10] J. Lewis, J. Rossi And A. Weitsman, On the growth of subharmonic functions along paths, Ark. Mat. 22 (1984), 109-119.

[11] J. Rossi And A. Weitsman, The growth of entire and harmonic functions along asymptotic paths, Comm. Math. Herv. 60 (1985), 1-14; its correction, ibid. 1516.

[12] J. Rossi, Second order differential equations with transcendental coefficients, Proc. Amer. Math. Soc. 97-1 (1986), 61-66.

[13] L.-C. Shen, Solution to a problem of S. Bank regarding the exponent of convergence of zeros of the solutions of a differential equation $f^{\prime \prime}+A f=0$, Kexue Tongbao 30 (1985), 1581-1585.

[14] M. N. M. TALPUR, A subharmonic analogue of Iversen's theorem, Proc. London Math. Soc. (3) 31 (1975), 129-148.

[15] M.N.M. TALPUR, On the growth of subharmonic functions on asymptotic paths, Proc. London Math. Soc. (3) 32 (1976), 193-198.

[16] N. Toda, On a modified deficiency of meromorphic functions, Tôhoku Math. J. 22 (1970), 635-658.

[17] M. Tsuji, Potential theory in modern function theory, Maruzen, Tokyo (1959).

\author{
Department of Mathematics \\ Nagoya Institute of Technology \\ Gokiso, Showa-ku, Nagoya 466 \\ JAPAN
}

